\title{
Relatos de pais de crianças com autismo sobre a rede de apoio formal: aspectos da elaboração parental do diagnóstico
}

\author{
Márcia Rejane Semensato* \\ Cleonice Alves Bosa**
}

\section{Resumo}

Este artigo apresenta evidências sobre aspectos que participam da elaboração parental do diagnóstico de autismo, especialmente sobre a rede de apoio. Esta rede tende a ser considerada, no relato dos pais, como essencial na reestruturação familiar, e é representada, principalmente, pelos profissionais e serviços de saúde. Acredita-se que estas crenças são determinantes na forma como os pais lidam com o diagnóstico e nas escolhas que fazem para o filho e para a família, como por exemplo, escolas e intervenções clínicas. Assim sendo, realizou-se uma Análise de Conteúdo em seis entrevistas de pais de crianças que tiveram diagnóstico de autismo. Estas entrevistas exploravam a história familiar destes casais e sua vivência a partir do diagnóstico do filho. Os resultados apresentam as crenças parentais relacionadas ao senso de amparo ou desamparo, na relação com os profissionais e serviços envolvidos no atendimento ao filho, desde a avaliação até a comunicação do diagnóstico e intervenções. Os resultados evidenciaram que a relação com os profissionais pode ser vista como uma aliança ou como fonte de estresse.

Palavras-chave: Autismo; Crenças; Rede de apoio.

\footnotetext{
* Doutorado em andamento em Programa de Pós-Graduação em Psicologia da Universidade Federal do Rio Grande do Sul (UFRGS). Porto Alegre, Rio Grande do Sul, Brasil.

** Professora Doutora da Universidade Federal do Rio Grande do Sul, Instituto de Psicologia, Departamento de Psicologia do Desenvolvimento e da Personalidade. Porto Alegre, Rio Grande do Sul, Brasil.
} 


\title{
Reports of parents of children with autism on the formal supporting network: aspects of the parental diagnostic elaboration
}

\begin{abstract}
This article presents evidence about aspects that participate in the process of elaborating the diagnosis of autism by the parents, especially about the supporting network. This network tends to be one of the essential aspects considered by the parents in the restructuring the family and it is represented primarily by professionals and health services. It is presumed that these beliefs are important in the way that parents deal with the diagnosis and the choices they make for the child and the family, for example, schools and clinical services that are chosen. Therefore, a Content Analysis was performed on six interviews of parents of children who have had a diagnosis of autism. These interviews explored the family history of these couples and their experience since the diagnosis of the son. The results showed the parental beliefs related to the sense of support or helplessness in relationship with professionals and child care related services, since the diagnostic evaluation up to the communication of the diagnosis and interventions. These results presented evidence that the relationship with the professionals can be seen both as an alliance and as a source of stress.
\end{abstract}

Keywords: Autism; Beliefs; Support network.

\section{Introdução}

Oautismo tem comocritérios diagnósticos a presença de comprometimento nas interações sociais recíprocas e nas modalidades de comunicação, bem como interesses e atividades restritas, estereotipadas e repetitivas, manifestados até os trinta e seis meses de idade (DSM IV TR, 2002). Tendo em vista a extensão e qualificação dos comprometimentos das pessoas com autismo, essa síndrome tende a ser considerada um estressor potencial. Isto porque a família necessitará uma revisão de expectativas de como a vida seria, bem como da organização e rotinas da família. Essa revisão, tanto de aspectos subjetivos quanto objetivos é parte da elaboração do diagnóstico pelos pais, o qual precisará ser reformulado em diversos momentos do ciclo vital, tanto familiar, quanto do filho. O processo de elaboração diagnóstica passa pela interpretação que o casal de pais atribui e constrói acerca deste diagnóstico do filho. Isto inclui as crenças sobre profissionais envolvidos, tanto no processo diagnóstico, como na comunicação do diagnóstico e nas intervenções, sejam estas educacionais ou clínicas ou em serviços de saúde e escolares. 


\section{A elaboração do diagnóstico de autismo pelos pais}

A investigação sobre o processo de elaboração do diagnóstico de autismo, realizada por Milshtein, Yirmiya, Oppenheim, Koren-Karie e Levi (2010) e por Oppenheim, Koren-Karie, Dolev e Yirmiya (2010) sugeriu que a elaboração parece ser um atributo mais dos pais do que das características das crianças com autismo. Os resultados demonstraram que uma resolução do diagnóstico não estava associada com a severidade dos sintomas da criança, mas com a maior sensibilidade das mães com suas crianças e maior grau de otimização da reestruturação familiar e atendimentos do filho. Algumas pesquisas sobre o tema também concluíram que o diagnóstico requer um luto para a sua elaboração (BARNETT et al.,2003; MILSHTEIN et al., 2009; OPPENHEIM et al., 2009). Isso significa uma nova representação de si e da criança no pós-diagnóstico, congruente com a realidade atual de ter uma criança com necessidades especiais. De acordo com Marvin e Pianta (1996), outros aspectos importantes na resolução do diagnóstico por parte dos pais é a capacidade de reduzirem o foco nas questões sobre as causas do diagnóstico do filho e de se mostrarem mais orientados ao presente e ao futuro.

Uma das formas de compreender essa elaboração são as crenças parentais, mais especificamente o sentido atribuído às vivências (WALSH, 2006). As crenças influenciam as respostas emocionais, decisões e ações das pessoas, permitindo aos membros da família fazer sentido de suas situações de crise. As crenças parentais sobre o autismo (ex.: etiologia, diagnóstico e tratamento) são influências na forma com que os pais vão lidar com as crianças e compreender seu comportamento, bem como na escolha do tratamento para o filho, nas decisões para o futuro e no planejamento familiar (HARRINGTON, et al., 2006; HERBERT; KOULOUGLIOTI, 2010, KING, et al., 2009; DARDENNES, et al. 2011; WANG, et al., 2011). Estas pesquisas ressaltam o reflexo das crenças no impacto do diagnóstico e a importância da sua investigação ao elaborar planos de atendimento para a criança com autismo e seus pais.

\section{Elaboração do diagnóstico: comunicação com profissionais e serviços}

Há várias evidências empíricas sugerindo que clientes que trabalham com profissionais da saúde com boas habilidades comunicativas interpessoais se beneficiam em termos psicológicos (satisfação com o serviço) e comportamentais (adesão ao tratamento), além de melhora dos índices de saúde (DICKSON; HARGIE; MORROW, 2003). A comunicação do diagnóstico parece ter um impacto importante na forma que a família vai lidar com o mesmo (SHARF; VANDERFORD, 2003; STREET JR., 2003). Assim sendo, essas investigações procuram entender os sentidos que os pacientes e sua família dão à doença, enquanto processos que afetam seu relacionamento e identidades. No caso do autismo, uma vez que o diagnóstico é comunicado, é importante orientar os pais sobre recursos que os auxiliem a desenvolver um senso de controle (SCHULMAN, 2002; ROLLAND, 2001), tais como busca da compreensão das manifestações comportamentais peculiares do autismo no seu próprio filho e estímulo a esclarecer as dúvidas que tenham. Outras formas de estimular o senso de controle são leituras sobre indivíduos com autismo e sua 
família e auxílio na busca de apoio e conforto, por exemplo, contato com outros pais de crianças com autismo (SCHULMAN, 2002).

Um aspecto importante para os profissionais compreenderem é a avaliação que a família faz do estressor e da implicação da dificuldade da criança para o seu desenvolvimento futuro e para a vida familiar (MILSHTEIN, et al., 2010). Em termos gerais, uma das formas de lidar com doenças ou outras condições que afetem as pessoas, passa pela elaboração de um significado para as experiências da vida, como o atribuído pelos pais ao autismo. Esse significado é baseado em crenças familiares (KLEINMAN, 1980; ROLLAND, 2001, WALSH, 2006). As crenças familiares, portanto, auxiliam na compreensão do significado de condições tal como o autismo para a família. Na abordagem de Walsh, por exemplo, o sistema de crenças é considerado um processo essencial para compreensão de como as famílias desenvolverão seus recursos frente a situações adversas. Partindo destes pressupostos, o atual estudo visou a investigar as crenças dos pais sobre o papel dos profissionais e dos serviços clínicos e educacionais no processo de elaboração do diagnóstico de autismo do filho desde o processo diagnóstico até o momento da entrevista, passados alguns anos após a comunicação formal deste.

\section{Método}

Este estudo utilizou entrevistas do banco de dados do projeto de "Coparentalidade"1 realizadas com seis casais que possuem um filho com diagnóstico de autismo há pelo menos dois anos, na época da entrevista, com idades entre cinco e dez anos. Os casais eram coabitantes e ambos os cônjuges eram os pais biológicos. As entrevistas utilizadas foram realizadas com base no Roteiro para Entrevistas sobre coparentalidade (NIEPED, 2004; 2008, investigando tópicos sobre a família, características individuais e do casal, conjugalidade, coparentalidade e parentalidade, bem como as ideias sobre o autismo e sobre o filho e a descrição de como foi essa experiência desde o momento do processo diagnóstico até os dias atuais. Essas entrevistas foram realizadas em estudos do NIEPED (SEMENSATO, 2009; SIFUENTES, 2007). Para a análise dos dados foi utilizada a Análise de Conteúdo (BARDIN, 2008; LAVILLE; DIONNE, 1999) das seis entrevistas utilizando como base o conceito do sistema de crenças, extraído da literatura sobre resiliência familiar (WALSH, 2006). Essas crenças foram investigadas em diversos aspectos relativos ao Autismo, ao Filho e ao Casal. Para este artigo focalizou-se as crenças sobre os profissionais e serviços de saúde e educacionais que participam da vida destas famílias em função do diagnóstico de autismo do filho. Os aspectos relacionados à elaboração parental do diagnóstico de autismo do filho foram selecionados tendo em vista a sua importância atribuída no relato dos pais.

\section{Apresentação e discussão dos resultados}

Os resultados são apresentados e discutidos com base nas categorias temáticas das crenças parentais acerca dos profissionais e dos serviços. No caso das crenças e concepções sobre os profissionais, estas são apresentadas tanto no processo diagnóstico, quanto nos atendimentos clínicos como, por exemplo, psicólogo, médico, 
fonoaudiólogo e nos atendimentos educacionais, representado principalmente pelos professores. Também são apresentadas as crenças e concepções sobre os serviços, incluindo serviços de saúde, representados pelas clinicas de atendimento e serviços educacionais, representados pelas escolas. Os relatos sobre os profissionais e os serviços são apresentados em termos do que despertou um senso de amparo ou de desamparo, tendo em vista a elaboração parental do diagnóstico. A seguir, apresentase a matriz de categorias:

Tabela: Crenças dos pais sobre os profissionais e serviços relacionados ao filho

\begin{tabular}{|c|c|c|}
\hline Crenças sobre os Profissionais & Processo diagnóstico & $\begin{array}{l}\text { Senso de amparo: } \\
\text { - Conhecimento sobre autismo. } \\
\text { - Habilidade com famílias na } \\
\text { comunicação do diagnóstico }\end{array}$ \\
\hline & $\begin{array}{l}\text { Atendimento clínico e } \\
\text { Educacional }\end{array}$ & $\begin{array}{l}\text { Senso de desamparo: } \\
\text { - preconceito } \\
\text { - falta de conhecimento sobre } \\
\text { autismo } \\
\text { Senso de amparo: } \\
\text { - no trato com as famílias (p. } \\
\text { ex.: empatia) } \\
\text { - no trato com as crianças (p. } \\
\text { ex.: afetividade, habilidade } \\
\text { específica para lidar com } \\
\text { crianças com autismo) } \\
\text { - trabalho em equipe }\end{array}$ \\
\hline & & $\begin{array}{l}\text { Senso de desamparo: } \\
\text { - falta de disponibilidade de } \\
\text { horário } \\
\text { - falta de competência e } \\
\text { habilidade (p. ex.: habilidades } \\
\text { comunicativas) }\end{array}$ \\
\hline $\begin{array}{l}\text { Crenças sobre os serviços } \\
\text { Educacionais e de Saúde }\end{array}$ & \multicolumn{2}{|c|}{$\begin{array}{l}\text { Senso de amparo: } \\
\text { - atendimento clínico em um só local (para as crianças e os pais) } \\
\text { - boas expectativas do desenvolvimento do filho em escola inclusiva } \\
\text { - escola que queria receber os pais e o filho } \\
\text { Senso de desamparo: } \\
\text { - falta de oferta no setor público se o caso não for muito sério } \\
\text { - temor pela segurança do filho na escola }\end{array}$} \\
\hline Recursos familiares & \multicolumn{2}{|c|}{$\begin{array}{l}\text { Rede de apoio } \\
\text { Senso de amparo: apoio familiar, apoio formal, profissional, apoio } \\
\text { de empregadores; apoio da escola e de outros pais em semelhante } \\
\text { situação. }\end{array}$} \\
\hline
\end{tabular}


Nos relatos, o Processo Diagnóstico foi considerado como as crenças dos pais no período desde o início da busca de respostas ao que percebiam de diferente do filho, incluindo visitas à escola, pediatra, psicólogo, neurologista. Isto porque, de acordo com o relato de vários casais, a "caminhada" rumo ao diagnóstico do filho iniciou na primeira "estranheza". Nas Crenças Sobre os Profissionais, no processo diagnóstico, o senso de amparo dos pais foi relacionado ao conhecimento dos profissionais sobre autismo e uma relação de confiança entre estes e a família. Foi ressaltada, pelos pais, a importância do conhecimento do desenvolvimento típico e atípico pelo profissional que lida com crianças e a capacidade de observação das peculiaridades de algumas crianças (ex.: diferenças das crianças com suspeita de autismo). Esses aspectos foram retratados por pais como fatores que melhoram o prognóstico no tratamento do filho, pois levam à identificação e consequente intervenção precoce do autismo. Outro aspecto retratado como fundamental na opinião dos pais, para a forma que inicia a elaboração do diagnóstico, é a habilidade dos profissionais de realizar a comunicação do diagnóstico sem, por um lado apenas rotular o que a criança apresenta ou, por outro lado, evitar usar a terminologia diagnóstica (o "termo" autismo). Os resultados também revelaram que a comunicação do diagnóstico é de fato um processo gradual para que o que é informado aos pais seja significado e, desta forma, compreendido. Nãoé, portanto, um único momento no qual os pais recebem a comunicação diagnóstica e orientações vagas sobre o devem fazer. A comunicação de um diagnóstico de um problema crônico requer boa habilidade de comunicação pelo profissional (DICKSON et al., 2003; SHARF; VANDERFORD, 2003; STREET Jr., 2003). A comunicação empática, de acordo com Platt e Keller (1994), é uma habilidade que pode ser ensinada e aprendida e envolve compreender os sentimentos e ideias dos envolvidos, sejam pacientes, familiares e profissionais. A comunicação do diagnóstico e a informação deste são processos interligados, mas também diferenciados. Nesse sentido, Oliveira, Oliveira, Gomes e Gasperin (2004) ressaltam que a informação envolve um parecer dado, cabendo à pessoa aceitar ou não. A comunicação permite à pessoa ter a informação, usar sua experiência para avaliar a informação e compreender que sentido isso faz para si.

O senso de desamparo em relação aos Profissionais, no processo diagnóstico, foi ligado à crença dos pais de que suas preocupações iniciais não são "levadas a sério", principalmente pelos pediatras, os primeiros procurados pelas famílias quando percebem algo diferente com o filho: “...a gente começou a procurar atendimento, só que era muito difícil,... porque ninguém dizia nada pra gente e as pessoas pareciam... assim que até fazia às vezes pouco caso" [M2]. Alguns pais também relatam sentir-se abalados pelo temor de serem responsabilizados como incompetentes pelos conhecidos ou pelos profissionais. Esse temor do estigma tende a estimular as famílias a se isolar dos demais (LAMANA; RIEDMAN, 2009). O senso de desamparo também foi representado por crenças de desconfiança em relação aos profissionais que escondem as informações dos pais (ex.: evitando dizer que é autismo mesmo que pais já tenham perguntado), a falta de conhecimento sobre autismo, principalmente no setor público (profissionais pouco esclarecidos sobre o que é típico do autismo), bem como o fato do conhecimento atual não responder a muitas dúvidas sobre o desenvolvimento da criança com autismo, a existência de poucos serviços especializados e o pouco contato dos familiares com informação acessível, conforme ilustra o relato a seguir: 
Eu sabia que (o autismo) era alguma coisa relacionada a... algum problema na cabeça. Mas não sabia os sintomas, o que que ele podia fazer, o que não podia fazer. Daí quando eu li num dicionário não lembro lá o que dizia,.. explicava assim... como um médico explica pra ti daí na... daí tu não entende muito [P4].

A dificuldade de comunicação também foi relatada como um desinvestimento dos professores em relação ao filho: "E outra coisa... é nosso relacionamento com... com professores, não tem..." [P6]. Os pais temem que os professores não considerem seus filhos alunos que tem motivos para estar lá.

A avaliação foi considerada longa, independente da habilidade profissional. A duração, no entanto, posteriormente foi compreendida como o "começo de uma reestruturação da família" [M2] e o início do tratamento da criança. Nesse sentido, os pais relataram que o tempo foi importante para a elaboração do diagnóstico:

a gente sabia que alguma coisa era... só não sabia o que que era né... [P2]; ... mas naquele meio tempo ali já tinha assim, ah, ele tem característica, ah, ele é assim, mas a gente não pode afirmar, então a gente já foi se acostumando com a ideia, a gente já foi procurando saber o que que era, sabe, a gente já foi conversando, mexeu com a família [M2].

Os relatos parentais indicaram que se o processo diagnóstico adquire um significado maior que uma resposta de "tem ou não ao autismo", ele é favorável ao processo de bem-estar do casal e da família. Na literatura sobre o tema, várias pesquisas sugerem que alguns pais sentem alívio no momento da comunicação do diagnóstico (FLEISCHMANN, 2004; HUTTON; CARON, 2005; MANSELL; MORRIS, 2004; MILSHTEIN et al., 2010; OPPENHEIM et al., 2009), pois percebiam que havia algo diferente acontecendo com a criança já há algum tempo.

O fato de este processo conter múltiplas avaliações foi considerado desfavorável no sentido de envolver muitos profissionais e muitos exames, o que gera desgaste - emocional, físico e financeiro às famílias. Por outro lado, a avaliação diagnóstica é sentida como necessária, até porque é considerada como uma forma de estabelecer um senso de controle e, conforme mencionado anteriormente, como o início da intervenção com o filho. A experiência com os profissionais e com os sistemas de serviço, no diagnóstico e na comunicação, deste à família, foi investigada por Fleischmann (2004), Hutton e Caron (2005) e Osborne e Reed (2008). Os sentimentos descritos pelos pais incluem, além do alívio, choque, culpa, raiva, esperança, sensações de perda e luto (FLEISCHMANN, 2004; HUTTON; CARON, 2005).

Nas Crenças sobre os Profissionais envolvidos nos atendimentos clínicos e educacionais o senso de amparo no trato com as famílias foi relacionado à habilidade daqueles ao lidar com a família e saber trabalhar com os pais as expectativas sobre o autismo e potencialidades do filho (ex.: que "não tem cura, mas tem tratamento" [P2]). O senso de amparo também foi expresso pela capacidade de troca de informações do 
profissional com a família durante o tratamento e a empatia, representada pelo saber "escutar e entender" [Pl]. Os profissionais também são fonte de amparo no tratamento pela habilidade para lidar especificamente com crianças com autismo e por gostar de crianças, o que, na concepção dos pais, é relacionado a melhores resultados:

[...] a nossa maior preocupação, assim, é...que fosse funcionar... aquelas pessoas, que não revelam afetividade pela criança, a gente não gosta muito, parece uma certa inadequação, assim, porque eu acho que a pessoa pra tratar com, pra cuidar de criança especial, ela tem que, tem que gostar de criança especial [P5].

A capacidade de trabalho em equipe pelos profissionais foi reconhecida para o sucesso do tratamento e como uma fonte de amparo para os pais. Esta capacidade remete à importância da rede de apoio profissional para a família e à importância da interdisciplinaridade, ou seja, na voz dos pais, a possibilidade dos "profissionais trabalharem conjuntamente" [P5]. Isto foi relacionado por eles a uma redução do desgaste da comunicação com diversas pessoas separadamente. O senso de amparo no tratamento também foi retratado pela aceitação do profissional pelo filho:

Tinha que ser um profissional... cuja a competência a gente pudesse ter alguma indicação e que ele gostasse [P5]. É, se ele não gostar pode ser a pessoa mais bem indicada do mundo, não adianta... não funciona [M5].

Por outro lado, o senso de desamparo, em relação aos profissionais, no tratamento do autismo do filho, foi relacionado à ideia da falta de disponibilidade de horário, a poucos profissionais habilitados para o tratamento do autismo, e má qualidade da comunicação dos profissionais com as famílias e entre si. Pais relataram temer que profissionais que atendem o filho também lhes atribuíssem culpas.

Quanto aos Serviços, foram relatadas crenças e concepções acerca das clínicas, das escolas especiais e das escolas inclusivas. O senso de amparo foi expresso principalmente pela existência de atendimento em um só local para as crianças e os pais, pois as famílias se sentem mais amparadas quando há um profissional que atua como uma referência e não apenas uma equipe em que várias pessoas nem sempre se comunicam entre si:

falta isso, algum lugar, assim, onde tu puder, tipo um centro integrado, sabe? Onde tu pudesse chegar lá, assim, atendessem a criança, atendessem os pais, tivesse orientação, sabe? Pudesse acompanhar... [P5].

O impacto dos serviços de saúde nas famílias, no caso de doenças crônicas é contemplado como muito importante no modelo de adaptação psicossocial de Bradford (1997). Nesse modelo, o impacto dos serviços de saúde é compreendido na sua interação com as crenças sobre saúde e os padrões de interação e comunicação familiar. 
As escolas são vistas como fonte de apoio quando profissionais da escola tem comunicação com os pais. A ida do filho à escola foi vista como muito benéfica para seu desenvolvimento: Tá bem sociável de lidar. muito por causa da escola, né? que ele aprendeu muita coisa lá, né? ele não fica, já não fica tão dependente, né? da gente. Porque ele já tá falando, já, né? já pede alguma coisa... já não precisa fazer, conseguiram lidar com essas dificuldades? [P4]. A escola tende a ser escolhida pelos pais por diversos aspectos reunidos desde o pedagógico, instalações e capacidade de acolher a família e a criança: Nós já fomos a duas escolas, nessas duas escolas nós fomos juntos... a gente tem que ver...muito além do pedagógico, né? porque a gente ta só procurando escolas que tenham a proposta inclusiva, né?... a gente ta... olhando a afetividade da escola... as ideias dos professores [M5].

Existe uma expectativa que o filho possa frequentar a escola, não só a escola especial: "a gente ainda espera...que ele possa...frequentar uma escola regular que tenha inclusão [P]". De fato, os pais que tiveram esta experiência, em geral, avaliam que a frequência à escola teve um crescimento de impacto no filho, como referido anteriormente. Por outro lado, os pais relatam que os filhos são 'recebidos' na escola, mas que gostariam que eles fossem mais verdadeiramente aceitos [P5]. Eles relataram que gostariam de chegar a uma escola e escutar, por exemplo: "sim, nós queremos receber seu filho aqui; nós nos sentimos seguros de receber um autista aqui... na nossa escola" ou "Nós já temos experiência com autismo e foi muito boa" entende?[P5]. Os pais relatam que algumas escolas chegam a ser inclusivas e não sabem:

embora a direção da escola ainda não admita ser inclusiva, mas pra nós ela é inclusiva, porque se o João é um autista e está numa escola regular, normal, pra nos é uma inclusão...o beneficio que a gente quer a gente tá atingindo, então deixa assim, né? [Ml].

Por outro lado, os pais, cujas crianças ainda estão na educação infantil temem a transição da troca para a escolarização formal, pois os filhos tendem a ficar bem nas escolas de educação infantil: as crianças lá, nessa idade pelo menos ali da creche, que, que eles são bem afetuosos e que gostam do Pedro, que ajudam o Pedro nas rotinas e tal, enfim, ele é bem aceito... minha preocupação... é o dia que tiver numa outra escola, né? que ele tenha essa outra discriminação ou que... né? isso aí eu não vou querer permitir assim [M6].

As instalações escolares são muito importantes não só no aspecto pedagógico, mas porque alguns pais temem pela segurança do filho frente a outras crianças:

eu tenho muito medo dos banheiros... um banheiro de escola, se vai um menino de oitava séria e uma criança de jardim usar o mesmo banheiro eu acho meio perigoso, porque eu não conheço as outras famílias[M].

Outros pais relatam a sua dificuldade de aceitar que o filho possa se adaptar bem na escola: "Daí eu me preocupava com o guri, não sabia se ele comia ou não, e o que. Tanto que eu nem mandava pra almoçar lá,... até agora mesmo, assim eu já nem mandava...” [M4]. 
Na rede de apoio, o senso de amparo foi expresso pela existência de amparo na família extensiva e pela capacidade de mobilização, principalmente da rede de apoio formal, pois os profissionais e serviços foram considerados importantes recursos para as famílias. A rede de apoio formal foi relatada como essencial desde o período do aparecimento dos sintomas até o momento atual (da entrevista). A mobilização de outros recursos envolveu a informação e o conhecimento. Nesse sentido, o senso de amparo foi relatado pela busca do conhecimento do que se passava com o filho, pelo contato com leituras relacionadas ao tema, pela participação em grupos, palestras e conversas com profissionais e entidades, pela procura de direitos legais para famílias de crianças com autismo, tal como a redução de carga horária profissional. Além disso, o contato com outras famílias que têm filhos em situação semelhante pode trazer auxílio na forma de lidar com a criança com autismo. Semelhantes resultados foram encontrados em um estudo com grupos de pais de crianças com autismo (SEMENSATO; SCHMIDT; BOSA, 2010) Outras fontes de apoio relatadas pelos pais são "pessoas jurídicas" que empregam familiares (ex.: apoio financeiro a fim de custear educação e saúde) e a escola, considerada uma grande fonte de apoio, pois, de acordo com os casais, são pessoas que conhecem bem o filho.

Os Recursos Familiares foram relatados com ênfase nos comprometimentos quando há dificuldade de mobilização da rede institucional/profissional (ex.: espera passiva de vaga para filho na escola), pouca disponibilidade de rede de apoio familiar, baixa mobilização de rede de apoio familiar, família nuclear não cooperativa - não trabalha junto, falta de amigos (solidão social do casal). De fato, na opinião de Lamana e Riedman (2009), quando as famílias se sentem estigmatizadas, tendem a se afastar de recursos. Assim sendo, as comunidades virtuais passaram a ser muito importantes: ali os pais podem ter informações de peritos e contato com outras famílias que estão vivendo situação semelhante, de forma que podem evitar a sua sensação de preconceito. Por outro lado, isso ressalta a importância de os profissionais estarem disponíveis e atentos, a fim de auxiliar as famílias a "filtrar" as diversas informações disponíveis na Internet para que esses recursos sejam potenciais e não fonte de desamparo.

Pelo exposto, compreende-se que a participação dos profissionais como facilitadores que acompanhem este processo pode ser de grande auxílio na elaboração do diagnóstico pelos pais. A elaboração dos pais tem consequências para a vida da criança e da família. Isso porque a tendência a adquirir um senso de controle sobre a situação parece iniciar já nos primeiros meses e é parte de um desenvolvimento contínuo destes. De fato, a importância das crenças na forma que as famílias lidam com o diagnóstico vem sendo reconhecida por profissionais de diferentes áreas que trabalham com autismo, pois pesquisas sobre a influência das crenças de familiares no impacto do diagnóstico são foco atual do interesse de profissionais da área médica (geneticistas, pediatras, clínicos gerais), da enfermagem e da psicologia. Isso porque a compreensão destas crenças pode auxiliar a melhor planejar programas de tratamento para essas crianças (DARDENNES et al., 2011; HARRINGTON et al., 2006; MILLER, et al., 2012). Além disso, as crenças provém de um material a que, na clínica e na escola, os profissionais têm acesso possível: os relatos parentais. 
A busca de sentido para problemas de saúde das crianças é parte das preocupações familiares (BOTEGA, 2007) e, como tal, precisa ser escutada pelos profissionais. Além disso, é preciso estar atentos ao fato de as preocupações parentais sobre o diagnóstico de autismo serem diferentes dependendo do momento (GUINCHAT et al., 2012). As estratégias usadas para tal, no entanto, podem ser fonte de estranheza nas equipes, se os profissionais não compreenderem a elaboração como um processo com várias tentativas diferentes de fazer sentido a essa vivência. Compreender essas estratégias e as crenças propicia maior empatia para com os pais. Além disso, é possível que, ao tomar contato com essas crenças, os profissionais tendam a corrigi-las imediatamente em função do temor de ser um indicativo de não aceitação do diagnóstico. No estudo de Oliveira, et al. (2004), a experiência dos profissionais da medicina, na comunicação do diagnóstico de problemas crônicos, foi relacionada com ser mais empáticos, a falar menos sem ser evasivo, dar as informações de forma mais gradual e a escutar mais os pais.

\section{Considerações finais}

As crenças sobre os profissionais e serviços foram indicativos importantes na elaboração do diagnóstico por parte dos pais. Elas representaram formas de interpretar diversos aspectos do que viveram, incluindo as informações recebidas dos profissionais. Pretende-se que esse conhecimento, que parte das concepções parentais, contribua para acompanhar o desenrolar e a facilitação do processo de elaboração do diagnóstico de autismo do filho pelos pais. Também se pretende que esse conhecimento possa ser utilizado na formação de profissionais que trabalham com famílias e na orientação de programas de intervenção para comunicação diagnóstica e intervenções posteriores com os familiares.

O entendimento da vivência destes pais também pode ser ampliado com pesquisas com triangulação de dados, envolvendo as crenças de profissionais que trabalham com essas crianças, tanto crenças sobre o autismo, como crenças sobre os pais ou familiares, como, por exemplo, de que modo profissionais lidam e compreendem as reações destes. Estas informações são relevantes para considerações sobre intervenções possíveis e para compreender a vivência destas famílias de forma mais integral. Melhorar a comunicação dos profissionais com os pais pressupõe poder escutar sua vivência a partir desses processos, e não exclusivamente a partir dos sintomas do filho.

\section{Referências}

AMERICAN PSYCHIATRIC ASSOCIATION: DSM - IV- TR. Manual diagnóstico e estatístico de transtornos mentais, 2004.

BARDIN, L. Análise de Conteúdo. Lisboa: Edições 70. (Original published in 1977), 2008.

BARNETT, D., Clements, M., Kaplan-Estrin, M.; Fialka, J. Building new dreams: supporting parents' adaptation to their child with special needs. Infants;Young Children, v.18, n.4, p. 295-307, 2003. 
BOTEGA, N. Prática psiquiátrica no hospital geral: interconsulta e emergência. Porto Alegre: Artmed, 2007.

BRADFORD, R. Children, family and chronic disease: psychological models and methods of care. London: Routledge, 1997.

DARDENNES, R., et al. Treating the cause of illness rather than the symptoms: parental causal beliefs and treatment choices in autism spectrum disorder. Developmental Disabilities, v. 32, n. 3, p. 1137-1146, 2011.

DICKSON, D.; HARGIE, O.; MORROW, N. Communication skills training for health professionals. London: Chapman and Hall, 2003.

FLEISCHMANN, A. Narratives published on the Internet by parents of children with autism: what do they reveal and why is it important? Focus on Autism and Other Developmental Disabilities, v. 191, p. 35-43, 2004.

GUINCHAT, et al. Very early signs of autism reported by parents include many concerns not specific to autism criteria. Research in Autism Spectrum Disorders, v.6, n.2, p. 589-601, 2012.

HARRINGTON, J. et al. Parental beliefs about autism: implications for the treating physician. Autism, v. 105, p. 452-462, 2006.

HUTTON, A. M.; CARON, S. L. Experiences of families with children with autism in rural New England. Focus on Autism and Other Developmental Disabilities, v. 203, p.180-189, 2005.

KLEINMAN, A. Patients and healers in the context of culture. Berkeley: University of California Press, 1980

LAMANNA, M.; RIEDMANN, A. Marriages and families: Making choices in a diverse society. Belmont, CA: Thomson, 2009.

LAVILLE, C.; DIONNE. J. A construção do saber: manual de metodologia da pesquisa em Ciências Humanas. Porto Alegre: Artmed, 1999.

MANSELL, W.; MORRIS, K. A survey of parents' reactions to the diagnosis of an autistic spectrum disorder by a local service: access to information and use of services. Autism, v.84, p.387-407, 2004.

MILSHTEIN, S., et al. Resolution of the diagnosis among parents of children with autism spectrum disorders: associations with child and parents characteristics. Journal of Autism and Developmental Disorders, v.40, p. 89-99, 2010.

OLIVEIRA, V. et al. Comunicação do diagnóstico: implicação no tratamento de adolescentes doente crônicos. Psicologia em Estudo, v. 91, p. 9-17, 2004.

OPPENHEIM, D. et al. Maternal insightfulness and resolution of the diagnosis are associated with secure attachment in preschoolers with autism spectrum disorders. Child Development, v. 80 , p. $519-527,2009$.

OSBORNE, L.A.; REED, P. Parents' perceptions of communication with professionals during the diagnosis of autism. Autism, v. 123, p. 309-324, 2008. 
PLATT, F.; KELLER, V. Empathic communication: a teachable and learnable skill. Journal of General Internal Medicine, v. 9, p. 222-226, 1994.

ROLLAND, J. Doença crônica e o ciclo de vida familiar. In B. CARTER; M. MCGOLDRICK, As mudanças no ciclo de vida familiar: uma estrutura para a terapia familiar. Porto Alegre: Artmed, 2001, p. 373-392.

SCHULMAN, C. Bridging the process between diagnosis and treatment. In R. GABRIELS; D. HILLS, Autism - From Research to Individualized Practice. London: Jessica Kingsley Publishers, 2002, p. 25-45.

SEMENSATO, M. S. Relações entre os scripts de apego individuais e compartilhados em casais com um filho com autismo. Dissertação (Mestrado em Psicologia do Desenvolvimento), Instituto de Psicologia, Universidade Federal do Rio Grande do Sul, Porto Alegre, RS, 2009.

SEMENSATO, M.; SCHMIDT, C.; BOSA, C. Grupo de Familiares de Pessoas com Autismo: Relatos de Experiências Parentais. Aletheia, v. 32, p. 183-194, 2010.

SHARF, B.; VANDERFORD, M. Illness narratives and the social construction of health. In T. L. THOMPSON, A. DORHY, K. MILLER ; R. PARROT, Handbook of Health Communication. London/New Jersey: Laurence Erlbaum Associates Publishers, 2003, p. 9-34.

SIFUENTES, M. As características da coparentalidade em pais de crianças com autismo em idade pré-escolar. Dissertação(Mestrado em Psicologia do Desenvolvimento), Universidade Federal do Rio Grande do Sul, Porto Alegre, RS, 2007.

STREET JR., R. Communication in medical encounters. In T. L. THOMPSON, A. DORHY, K. MILLER; R. PARROT, Handbook of Health Communication. London/New Jersey: Laurence Erlbaum Associates Publishers, 2003, p. 63-89.

WALSH, F. Strengthening Family Resilience. London: The Guilford Press, 2006.

\section{Notas}

'Aprovação no Comitê de Ética em Pesquisa da UFRGS (processo número 2060030483).

\section{Correspondência}

Cleonice Alves Bosa - Universidade Federal do Rio Grande do Sul, Instituto de Psicologia, Departamento de Psicologia do Desenvolvimento e da Personalidade. Ramiro Barcelos 2600, sala 110, Santana, CEP: 90035-003 - Porto Alegre, RS - Brasil.

E-mail: cleobosa@gmail.com - msemensato@gmail.com

Recebido em 09 de março de 2013

Aprovado em 17 de junho de 2013 
